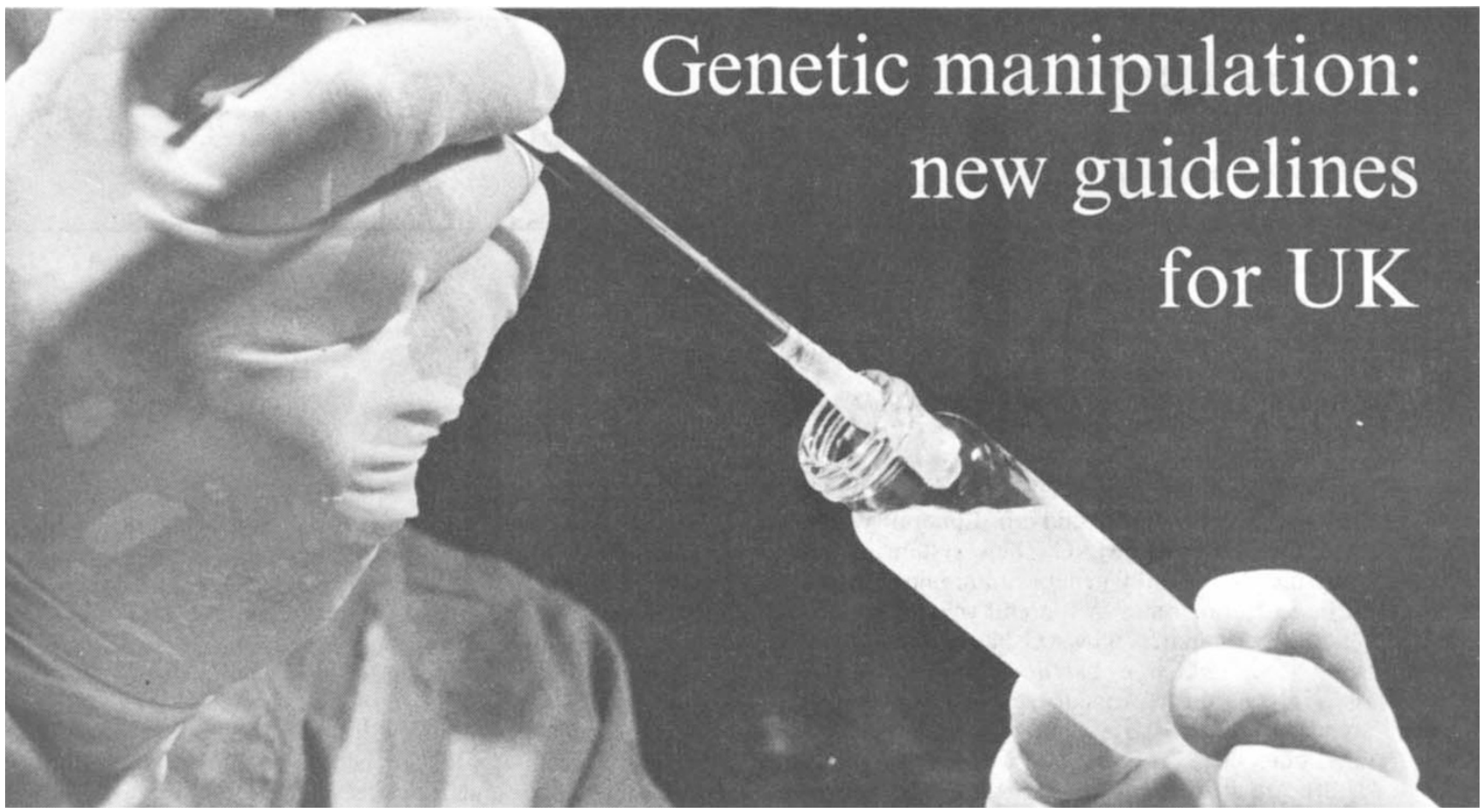

Britain's Genetic Manipulation Advisory Group (GMAG) is preparing to adopt a new approach to assessing the risks of 'genetic engineering' experiments. GMAG's statement -approved in principle at a meeting on 2 November-appears below. The scheme is based on an attempt to assign numerical risks to individual experiments as a product of three factors: the 'access factor', the 'expression factor', and the 'damage factor'. But the numbers are used only to rank the risks so that the experiments can be assigned consistently to the containment categories I to IV. Qualitative matters are also taken into account, for biology has not yet been reduced entirely to numbers.

The scheme will be used when there are enough data to employ it; otherwise assessment will revert to the old Williams 'phylogenetic' guidelines. Its great virtue appears to lie in its objective scientific basis and its consequent encouragement to research on risks.

GMAG's Sub-Committee on the Validation of Safe Vectors* and, more recently, an ad hoc Working Party on Guideline Criteriat, have been considering on GMAG's behalf the merits of an alternative approach to the categorisation of genetic manipulation experiments first outlined by Dr Sydney Brenner (MRC Laboratory of Molecular Biology, Cambridge). GMAG, having considered the enthusiastic advice it has received, is persuaded of the general soundness of the new system, and subject to the consideration of comment on this dis- cussion document and consultation with the Department of Education and Science and the Health and Safety Executive, hopes to introduce the new principles-to be used for the time being in parallel with the existing Williams Guidelines ${ }^{1}$ - early in 1979. Before refining the system, GMAG wishes to take into account the opinions of the scientific community, and it is therefore hoped that publication of the following will allow all concerned to make their views known to the Group $\ddagger$ In the very near future, GMAG will be arranging a meeting with users and with members of local Biological Safety Committees.

While seeing justification and opportunities for change, GMAG considers that there is no case for changing the general arrangements governing this area of research in the UK: the definition of genetic manipulation remains unchanged; the regulations $^{2}$ to notify work being done must apply; the roles of GMAG and HSE have been established; facilities for physical containment (categories I-IV) have been defined and substantial investment has gone into their construction. ${ }^{3}$ Proposals are put forward in this paper, however, which if implemented would lead to change in the scientific considerations upon which the categorisations of individual experiments are decided by GMAG.

At present, GMAG broadly follows the Report of the Williams Working Party in allotting experimental protocols to the four categories of physical containment. The chief conclusion of the arguments which follow is that those experiments for which it is possible to carry out an approximate risk assessment should where appropriate be given categorisations distinguished by the labels I*. II*, III* and IV*. (It must be emphasised that no change is implied in the specification of physical containment facilities, but only in the categorisation of certain experiments.) It is suggested that the two systems of categorisation should persist in parallel for as long as may be necessary. At this stage relatively few experiments can be given the new starred categorisations, but a steady transfer of experiments and classes of experiments from one system to the other should be possible.

\section{The Williams guidelines}

Although in the past two years GMAG has based its advice on the "suggested categorisations" of the Williams Report, there have been several occasions when it has heen necessary to modify or to extend that scheme. That this should have been so is not surprising, for the Williams Working Party reported when there had been very little practical experience of genetic manipulation. Moreover, the report explicitly acknowledged that its categorisations were likely to be found insufficient in some respects, and it

*Professor J. H. Subak-Sharpe (Chairman), $\operatorname{Dr} S$. Brenner, Professor D. C. Ellwood, Dr M. Fried, Dr M. Moss (Secretary). Professor K. Murray, Dr N. Murray, Professor M. H. Richmond, Dr P. W.J. Rigby, Dr R. Weiss, Dr N. Wilkie. Professor R. Williamson.

tMr I. Maddox (Chairman), Professor K. R. Dumbell, Professor M. H. Richmond, $\mathrm{Dr} R$. Weiss, $\mathrm{Dr}$ J. H. Morris (Secretary).

†ecretary, GMAG, at the Medical Research Council, 20 Park Crescent, London WIN $4 A L$. 
foresaw the need for refinement and substantial modification.

First, doubts have accumulated about the value of the criterion of phylogenetic relatedness as a measure of the possibility that genetic manipulations may be hazardous. A simple illustration will show the difficulty. According to the suggested categorisations, genetic elements from plants entail less relative hazard (to human beings) than genetic elements from higher organisms, yet it is also clear that the incorporation into the genome of a strain of $E$. coli viable in the human gut of a gene responsible for a plant immunogen (say the glycoprotein gluten from wheat germ) could be relatively more hazardous than a gene responsible for the production of, say, human globin. More generally, the bearing of phylogenetic relatedness on the potential dangers of experiments in genetic manipulation must be modified by the nature of the host and the vector; animal genes are, for example, less likely to retain their original functions when incorporated in the genomes of bacteria than in those of animal cells or viruses.

Second, the experience of the past two years has also shown that there is a need for a modification of the Williams prescription about the identity and the purity of transplanted genetic elements. Often the more relevant characteristic is whether the foreign gene is capable of being expressed for otherwise retaining its original function). Moreover, evidence has accumulated in the past two years to suggest that many of the genes of higher organisms are distributed as separate pieces among the genetic material of the donor organism, which decreases the likelihood that shotgun experiments in which pieces of random DNA are transferred to other (and especially bacterial) hosts will yield functional genes in the new genetic environment. On the other hand experiments in which genetic elements are obtained in potentially functioning form from functional molecules of messenger RNA. often regarded in the past two years as an assurance of their purity, may be in principle more potentially hazardous than the same genetic elements obtained directly from the genomic donor DNA. These statements are not. of course, of general application, but they are another reason to believe that individual cases should be dealt with separately.

Considerations of the host-vector system remain generally applicable. If there are hazards in any experiment in genetic manipulation, it will plainly be beneficial that a host organism carrying foreign genetic elements should be incapable of infecting human beings, and that the vectors used in the experi- ments should be specific to these host organisms. Appropriate use of "disabled" host-vector systems, offering "biological containment", will continue to be one of the essential features of certain types of recombinant DNA research.

\section{More objective methods}

In the circumstances, it is likely that all interested parties, scientists and the general public alike, would benefit if there were a more objective and even quantitative basis for assessing the risks of genetic manipulation. But it will be some time, perhaps several years, before biological understanding and quantitative data have accumulated sufficiently for such techniques to be applied to all proposals for experiments in genetic manipulation.

\section{Elements of risk assessment}

Assessment of the risk of experiments in genetic manipulation must follow a formal and comprehensive risk analysis, which is possible only when the biology of the host-vector system and of its interaction with the cells of organisms at risk is reasonably well known. At present the relevant biological data are not generally available, and many systems of potential value in genetic manipulation will therefore be excluded from the categorisation procedure now proposed. With the passage of time, however, it will be possible to include within the new basis for categorisation an increasing proportion of experimental proposals.

In general, risk analysis entails a consideration of the following steps.

(a) Escape from containment

The escape of organisms carrying foreign genetic elements from the containment specified for them is always a possibility, either because the facilities are not designed in such a way as to provide absolute assurance that organisms will not escape or because of some malfunction or accident. Thus it is proper to associate with each category of containment a specific probability $\left(C_{1}\right.$, where $i$ runs from $I$ to IV) that organisms will escape. The quantities $C_{i}$ have the units "probability per unit time per organism contained".

The ratios of these probabilities will be measures of the relative efficacy of the four different levels of containment, and there are at present only rough estimates of the ratio $C_{\mathrm{IV}} / C_{\mathrm{I}}$, the relative safety of the most stringent and the least stringent of the containment categories now in use. The ratio is likely to be less than $10^{-6}$ (corresponding to a factor of 100 for the interval between each category of containment) and may be less than $10^{-4}$. It is important that the true range of safety offered by the four categories of containment should urgently be defined more precisely. On present indications, the range of efficacy spanning the least and the most stringent categories of containment is comparable with the degree to which the viability of some host organisms is reduced relative to the wild-type strains; this points to questions for the future about the relative protection from conjectured hazard of physical and biological containment.

\section{(b) Persistence in the environment}

The most direct concern in the assessment of the risks of genetic manipulation is that organisms carrying foreign genetic elements will gain access to the tissues of laboratory workers, but it is important that those concerned with the safety of genetic manipulation should consider the posibility that, as an intermediate step, organisms may lodge and even replicate in the laboratory into which they have escaped (as, for example, in culture dishes). In this and other parts of the analysis, care must be taken to identify all the possible circumstances in which organisms carying particular foreign genetic elements may be relatively at an advantage, so that the genes concerned are amplified and not attenuated.

(c) Access to organisms at risk

Organisms which escape as aerosols may be inhaled by laboratory workers or ingested, thus gaining access directly to the nasal tracts, the lungs and the gut. It is important that unfamiliar routes of access should not be overlooked-for example, the effects on human lungs of the inhalation of $E$. coli organisms carrying genes capable of producing, say, insulin are at present unknown.

\section{(d) Replication}

The risk analysis of experiments in genetic manipulation differs from that of, say, the opcration of nuclear power plants in that agents which may potentially cause damage may in some circumstances be capable of replication. In the risk analysis of experiments in genetic manipulation, great care should therefore be taken to identify pathways that permit the replication of organisms carrying foreign genetic elements or which permit the transfer of the foreign genetic elements to other organisms which are capable of replication. One factor in a quantitative measure of a particular hazard would no doubt be provided by the duration and the intensity of the exposure of susceptible cels to organisms carrying potentially damaging genetic elements, and it is theoretically possible to calculate quantities such as these, given assumptions about the viability of manipulated organisms in the environments in which they lodge. Such formal calculations are however unlikely to be 


\section{Examples of the new categories}

- The cloning of pure mammalian globin genes in an approved disabled $E$. coli host-vector system in circumstances in which the globin genes are not expressed would be I*. For the time being, the use of non-disabled strains of $E$. coli (including $\mathrm{K} 12$ ) or the expression of the globin genes in a disabled host-vector system would place the experiment in category $\mathrm{II}^{*}$. The cloning of expressible globin genes in a non-disabled host-vector system would, for the time being, be a category III* experiment. The allocations to these higher categories are justified by the present lack of certainty that the secretion of mammalian globin in the body tracts (including lungs and the genitourinary tract) to which $E$. coli has access would be without risk. As this uncertainty is removed, lower categorisation may be considered appropriate.

Category $I^{*}$ is justified for the cloning of non-expressible globin genes in a disabled host-vector system on the following grounds-all three of the factors $A, E$ and $D$ are small (notionally less than unity). If it emerges that $D$ is small even when globin genes are expressed, the experiments provisionally allocated to II* and III* might be reduced in categorisation.

This experiment may therefore be considered typical of those destined for Category I*. It should be noted that two of the three factors afford a measure of biological protection, and that in spite of the apparent innocuousness of globin, GMAG would probably be unwilling to agree that an experiment of this kind should be undertaken with wild-type E. coli.

- The cloning of genetic elements from the genome of the mouse with the use of defective polyoma virus as a vector, and with cultured mouse cells as hosts (with appropriate helper virus to facilitate the replication of the virus and the expression of functionable genes) would also meaningful in present circumstances, so that more qualitative comparisons must at present suffice.

Great care must be taken to ensure that full allowance is made for the unavoidable incompleteness of present knowledge of the behaviour of foreign genetic elements in their hosts. Thus allowance must be made for the possibility that a "disabled" strain will revert (by genetic mutation) to the wild type, or that a foreign gene will be transferred (by the mobilisation of the plasmid) to wild-type bacteria. As things are, sufficient numerical data do not exist bearing on the chance that such events will occur, so that, for some time to come, researchers will have to rely on order-of-magnitude estimates of some of these quantities.

\section{The conjectured hazards}

Concern that genetic manipulation may entail hazards for living things stems from the possibility that foreign genetic elements may affect the normal functioning of particular groups of cells or the coordinated functioning of organisms at risk. Another of the impediments to formal and quantitative risk assessment that is likely to persist for some time is that of comparing the possible (but still hypothetical) biological consequences of foreign genetic elements with the damage done by naturally occurring disease. Even so, a tentative listing of some of the possible interactions between foreign genetic elements is suggestive of some of the points to which researchers should direct attention.

(i) Cell death

Cells may be killed when they are infected by viruses or when they are exposed to toxins produced by bacteria. Specific microorganisms and viruses often affect specific groups of cells in the body of a higher organism-poliomyelitis kills people because the poliomyelitis virus kills cells in the central nervous system when it has access to them, for example. In the analysis of the risks of genetic manipulation, the questions arise whether bacteria or viruses carrying foreign genetic elements are more virulent than the originals, whether the range of their specificity has been extended and whether bacteria carrying the intact genomes of pathogenic viruses make it possible for cells at risk to be exposed to potentially damaging viruses by unnatural routes.

(ii) Changes of genetic constitution The introduction of foreign genetic elements into human somatic cells could have a number of harmful consequences. The state of differentiation of the cell might be affected. Cryptic viral elements already included in the DNA of the cell might be mobilised, or the foreign genetic elements themselves might be incorporated in the genome of the intact cell, increasing the range of cryptic viral function embodied in the cell. The chief causes of anxiety on such grounds have so far been in connexion with the occurrence of cancer, but understanding is far from complete. Experiments in which animal viruses multiply in human cells (in tissue cultures) without killing the cells may deserve special attention.

(iii) Immunological consequences

There are several hypothetical mechanisms whereby untoward immunological reactions might be provoked by foreign genetic elements. Thus foreign genetic elements might be incorporated in the genome of a human cell in such a way that the product of that gene (possibly a protein) was expressed on the cell surface, thus exposing the cells affected to attack by the body's immunological defences; this may correspond to the mechanism of some naturally occurring autoimmune diseases. On the other hand, the introduction of foreign genetic elements might be accomplished in such a way that their presence

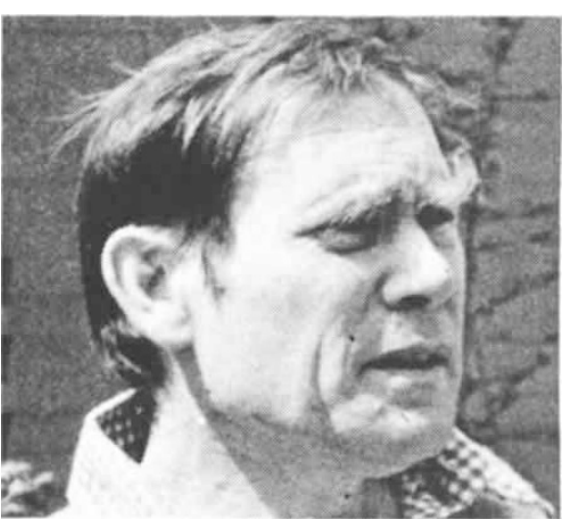

Sydney Brenner

simulates that of the normal components of body tissues, with the result that the range of the sensitivity of the immune system would be narrowed. In principle, it is also possible that some of the cells of the immune system are especially vulnerable to organisms carrying particular genetic elements.

(iv) Disturbances of endocrine function Many hormones are believed to exert their physiological effects by interacting with specific biochemical elements either on cell surfaces or in the nuclear DNA. Foreign genetic clements could in principle interfere with either of these reactions, but it is also the case that infection by bacteria carrying genes which direct the synthesis of human hormones and which are expressed and then secreted into the body could be damaging to the individuals concerned.

(v) Disturbances of cell metabolism Foreign genetic elements inadvertently introduced into somatic cells could in principle disturb the metabolism of these cells, changing their metabolic rate, the rate of cell division or even the process (still unknown) by which somatic cells age.

(vi) Consequences for microorganisms Experiments in genetic manipulation in which bacteria might artificially be en- 
be a category $I^{*}$ experiment. Formally, such experiments have much in common with those in which fragments of the E. coli genome are redistributed within the same organism with the help of lambda bacteriophage. In reality, however, the possibility that polyoma virus may replicate in cells other than those of the mouse cannot be entirely discounted (replication in rat cells has been demonstrated, while the possibility that polyoma might replicate in human cells has not been vigorously explored). Accordingly, the cloning of pure mouse genes in polyoma and cultured mouse cells is suggested as a category I* experiment, with mouse-genome shotgun experiments in category II* for the time being.

- Category $I^{*}$ will also include those experiments for which at present there is no substantial evidence of risk. The chief candidates of this kind are the experiments in which $E$. coli genes are cloned in $E$. coli organisms.

- If these illustrations are taken as a paradigm of experiments in category $I^{*}$, then it would follow that disabled E. coli carrying but capable of expressing the gene for, say, wheat germ gluten would be categorised $\mathrm{II}^{*}$ to allow for the possibility that at least some members of the population might react immunologically against the production of gluten in such a way. Other experiments that might be given the same categorisation on further consideration by the technical panel might include the incorporation of non-expressed interferon genes in $E$. coli $\mathrm{K} 12$, or expressed interferon genes in a more disabled strain of E.coli K12 (eg x1776). Shotgun experiments with mouse DNA cloned in polyoma virus would also be included.

- Similarly, and again on further examination by the technical panel, category III* might include disabled E. coli strains carrying expressed human growth hormone genes; the expression of cholera toxin genes in disabled strains of $E$. coli would remain in category $\mathrm{IV}^{*}$.

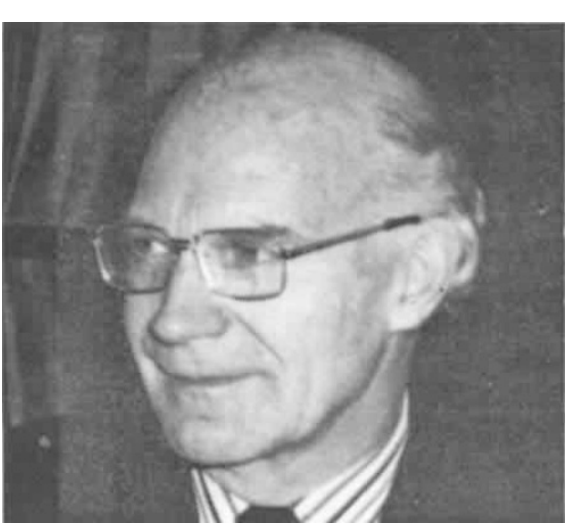

Sir Gordon Wolstenholme

dowed with resistance to antibiotics of medical or veterinary importance are potentially hazardous. Another early suggestion of a damage mechanism is the possibility that apparently innocuous organisms (such as the laboratory strains of $E$. coli $\mathrm{K} 12$ ) could be converted by genetic manipulation into infectious pathogens; relevant in this regard is the conclusion of a conference held at Falmouth (New Hampshire, USA) in 1977 that E. coli K12 at least cannot be converted to an epidemic pathogen. There are three kinds of reasons for this conclusion: the gentic basis of pathogenicity is complex and does not reside in a single gene. the wild-type strains of $E$. coli which have evolved are probably in some sense optimal for the ecological niches they occupy and the viability of laboratory strains of $E$. coli is so much less than that of wild-type bacteria that such organisms carrying pathogenic elements would probably be eliminated rapidly. This conclusion does not imply that individuals could not be damaged by $E$. coli carrying pathogenic elements such as the gene for cholera toxin, but merely that the production of an infectious pathogenic agent is improbable. There is a need for a consideration along the same lines of other organisms capable of infecting human beings.

(vii) Ecological consequences

Similar considerations apply to the interaction of foreign genetic elements and species other than human beings. To the extent that species were differently affected and that foreign genetic elements were widespread, ecological changes are in principle possible. Changes in the ecological balance of microorganisms are perhaps especially important.

For those assessing recombinant DNA experiments, this list should make it possible to single out those kinds of interactions between foreign genetic elements and cells which are likely to be widely regarded as potentially serious and damaging. Possible immunological and oncogenic consequences point to the need for a relatively high category of physical containment.

\section{Application in the immediate future}

In an ideal world, it would be possible to make numerical estimates of the risk of experiments in genetic manipulation by multiplying together the probabilities representing the various events $(a)$ to $(d)$ (see page 105), summing over all possible pathways leading from the escape of a genetically altered organism from its containment to the causation of a particular consequence for cells exposed and then multiplying by factors which take account of the costs of the damage that results. This is not now possible, however. Some may even argue that it will never be possible or necessary.

Nevertheless it is possible to use the framework of this formal risk assessment to rank the relative risks of some experiments in genetic manipulation, albeit in a somewhat qualitative manner. To that extent, the arguments first put forward by Dr Sydney Brenner in the GMAG Safe Vectors Sub-
Committee are a valuable means of moving towards a more objective system of categorisation.

What is now proposed is that experiments with which researchers are familiar should be allocated to the four containment categories on the basis of a qualitative and relative assessment of their risks; and that the potential risks of novel experiments should then be assessed in relation to these familiar paradigm experiments.

For this purpose, it is convenient (as Brenner has argued) to define a group of three factors which may be taken to represent the chance that events following the escape of genetically altered organisms into the environment may lead to particular conjectured hazards. If data were available, it would then be possible to think of calculating the risk of an experiment by multiplying the numerical values of the three factors together. For the time being, however, such a procedure would provide a spurious sense of precision and it is proposed that there should be a comparison (which necessarily entails the exercise of people's judgment) of the values of the three factors relative to those which obtain for the paradigm experiments.

Analysis might therefore proceed as follows. First, it is supposed that there is an escape of organisms carrying foreign genetic elements. The overriding element in the assessment of the risk of a particular kind of disturbance is the likelihood that the organisms or the genetic elements they carry will have access to susceptible cells of the target organisms. Thus there is defined an

- Access factor $(A)$. This is plainly a composite of factors representing the chance that escaped organisms will actually enter the human body, that they will survive there and that they will penetrate whatever membranes must be penetrated so as to reach the 
tissues containing susceptible cells. For many experiments, biological containment is dominant-for example the disabled strain of $E$. coli $\mathrm{K} 12$ known as $\chi 1776$ may be less viable than wildtype strains of $E$. coli by a factor of $10^{6}$.

In some experiments in genetic manipulation, the potential risk depends crucially on whether the foreign genetic element is able to express its normal function. This would, for example, be the case in an experiment in which the gene specifying the cholera toxin was incorporated in a plasmid of $E$. coli. Thus it is convenient to define an

- Expression factor $(E)$, which would be large (or notionally have the numerical value 1) if the foreign gene were efficiently translated into the protein product of the gene and if the products were then secreted from the altered organism, which would have a similar value in those circumstances (as for example in the carriage of a genetic element which can be integrated into the genome of target cells without being translated) but which would be small in those experiments in which steps had been taken deliberately to ensure that the gene would not be expressed.

Shotgun experiments require further consideration. The fear that "unknown" genetic elements might cause unknown and damaging changes in organisms at risk has been widespread for the past four years. The Williams Report suggested that experiments involving random lengths of DNA should be categorised somewhere in between the categonisation appropriate for genetic elements known to be damaging (eg cholera toxin) and those thought to be without risk. This conclusion remains valid, but knowledge of the genetic constitution of organisms is growing quickly, and for some it is now reasonably certain that a genetic element drawn at random is likely to be less of a relative hazard than one deliberately selected. This, for example, may be the case with many animal viruses, whose genetic structures appear to be so economical of DNA that it is hard to believe that a piece drawn at random would have physiological significance. Thus it is convenient to define a

- Damage factor $(D)$, which expresses the chance that a genetic element will cause damage. $D$ will be large (notionally, unity) if, for example, the host organism were known to contain a gene specifying a bacterial toxin; will be less if it is a genetic element drawn at random from the same bactcrial genome; and will be very small if the product of the gene is known to be without physiological effect on the cells of the target organism (as may be the case where the gene product is, for example, human globin).

It is proposed that, in the months ahead, GMAG should take steps to allow researchers (if they wish) to submit their proposals to GMAG with evidence which will allow GMAG to make an assessment of the three factors and advise on the new basis. Given the present incompleteness of biological understanding and the lack of data bearing on the numerical determination of these factors, it is clear that, for the time being, decisions will continue to depend on judgment rather than calculation. It is also clear that experiments using familiar systems will be the most susceptible to this alternative treatment. One advantage of following such a course of action will nevertheless be to encourage the accumulation of data bearing on the ultimately more objective assessment of risks.

\section{Proposals}

The particular recommendations that follow are designed so as to achieve the following objectives. First, the intellectual framework of risk analysis should be used as a means of further refining the self-consistency of GMAG's present procedures. Second, GMAG's procedures should remain flexible, and be capable quickly of being adjusted in the light of new knowledge. Third, new criteria for deciding on categorisations should be applied at the outset only to the systems with which researchers are most familiar, with the expectation that, with the passage of time, increasing numbers of experiments will be included.

It is therefore proposed that GMAG should take the following steps as soon as this may be convenient:

1. The present Williams categorisations will be retained, and GMAG's present methods of assessment will be retained for experiments whose relative hazards cannot be assessed in the manner outlined above. But researchers will also be invited to ask that experiments should be given a different categorisation than suggested by Williams if they have reason to believe that in their case the factors $A, E$ and $D$ would justify such a course. Similarly, GMAG should be able to require more stringent categorisation in appropriate circumstances.

2. GMAG will set up a technical panel to scrutinise such applications on the alternative basis and to report to GMAG, to collect data bearing on a more thorough understanding and evaluation of the factors $A, E$ and $D$, and to publish (through GMAG) to the scientific community advice on the continuing refinement of the procedure. Researchers might have to accept that, initially at least, these complementary procedures might entail some delay.
In the first instance, it would be for applicants to provide such empirical data as they can on which the technical panel's consideration of the three factors would be based. It is hoped, however, that the technical panel would be a powerful stimulus of the research needed to make risk assessment more objective and widely applicable. Refinement of present knowledge of the relative efficacy of the different categories of containment may be thought an urgent need.

3. The proposed system must be grounded by the allocation of some paradigm experiments to the four categories of containment.

4. It is proposed that for experiments of the kind referred to in the box (item (c)), and for others in which the genes of Bacillus subtilis and Pseudomonas aeruginosa are rearranged, it should be open to laboratories to provide a block notification of a programme of experiments to be carried out over a substantial period of time, say a year. A condition of the acceptance of such a notification will be the inspection of the laboratory by GMAG, an undertaking to work in category $I^{*}$ conditions and to submit a report at some required interval (not less than a year) providing a detailed $\log$ of all experiments carried out. Laboratories working to such a block notification would be required to inform GMAG of all new workers in the laboratory, and of the transfer of organisms to other laboratories; but the use of such organisms in recipient laboratories could be carried out under conditions of good microbiological practice (PHLS Handbook No. 4). In due course, this category of experiments without discernible risk will no doubt be extended.

\section{Acknowledgments}

The credit for urging on GMAG the risk assessment approach to genetic manipulation and for drawing it first to the attention of GMAG's Safe Vectors Sub-Committee goes to Dr Sydney Brenner, whose cooperation in developing the system into its present form is also warmly acknowledged. GMAG also wishes to thank the Guideline Criteria Working Party for their work in drafting the present paper, and innumerable other experts who have generously given their advice.

\section{References} 1 Report of the Working Party on the Practice of
Genetics Manipulation. HMSO, Cmnd 6600, Genet

2 Health and Safety (Genetic Manipulation) tions 1978. SI 1978, No. 752. First Report of the Genetic Manipulation .

Additional copies of this document are available at a price (including postage) of $25 p$ each, or $20 p$ each for orders of five or more. Send cheque, payable to Nature, to Promotion Manager, Nature, 4 Little Essex Street, London WC2. 